\title{
Tratamiento quirúrgico de cáncer pulmonar y valvulopatía cardíaca sincrónicos. Revisión de la literatura
}

\author{
Gildardo Cortés-Julián, 凶Juan Carlos Vázquez-Minero, Enrique Guzmán-de Alba
}

Instituto Nacional de Enfermedades Respiratorias Ismael Cosío Villegas, Ciudad de México.

Trabajo recibido: 02-III-2015; aceptado: 05-III-2015

\begin{abstract}
RESUMEN. Antecedentes: El tratamiento quirúrgico con intención curativa del cáncer de pulmón en pacientes con cardiopatía que también requieren de tratamiento quirúrgico, es un tema controversial dentro de la cirugía cardiotorácica. Se han descrito diferentes abordajes para dar solución a este problema ya que el orden de las intervenciones puede dar lugar a problemas de diversa índole. Gracias a la evidencia que aportan múltiples series de casos, se han podido extraer algunas conclusiones que son de utilidad clínica. Material y métodos: Revisión de la literatura publicada en PubMed. Resultados: Las escasas series muestran un incremento en la realización de ambas cirugías durante el mismo evento quirúrgico sin aumentar los riesgos perioperatorios en forma importante. Conclusión: No hay suficientes evidencias clínicas de buena calidad metodológica para recomendar o no las intervenciones sincrónicas. Los reportes de caso deben utilizarse con precaución.
\end{abstract}

Palabras clave: Cáncer de pulmón, tratamiento quirúrgico, valvulopatía cardíaca.

ABSTRACT. Antecedents: Curative intended surgical treatment of lung cancer, in patients with cardiac disease that is also amenable to a surgical procedure, is a controversial hot topic inside the cardiothoracic surgery. Since the order of the interventions could carry different problems during its performance, multiple approaches to this situations, have been described. Material and methods: Review of the literature published in PubMed. Results: There is a high volume of case reports that shows tendency towards an increasing performance of both procedures with the same anesthesia without a significative increase of the complications. Conclusion: There is a lack of enough clinical studies of good quality for give a recommendation, the case reports must be taken in count with reservation.

Key words: Lung cancer, surgical treatment, cardiac valvulopathy.

\section{INTRODUCCIÓN}

El cáncer de pulmón es actualmente la primera causa de muerte por cáncer de ambos sexos en nuestro país. ${ }^{1}$ Asimismo, se sabe que el tratamiento quirúrgico ofrece una sobrevida global de $32 \%$ a cinco años. Por el contrario cuando no se ofrece el mismo, la sobrevida disminuye hasta el $9 \% .{ }^{2}$ Uno de los factores de riesgo más importantes durante la cirugía, para presentar complicaciones que ponen en riesgo la vida de estos pacientes, es la presencia de cardiopatía descompensada. ${ }^{3}$ La viñeta clínica que se presenta a continuación es representativa de este problema, tal y como ha ocurrido en algunas ocasiones en nuestro servicio.

Paciente masculino de 69 años con antecedente de consumo importante de tabaco. Acude al hospital tres meses previos a su ingreso por disnea progresiva. Se realizó el diagnóstico de estenosis aórtica crítica candidato a reemplazo valvular. Durante el abordaje se documentó un tumor pulmonar de $7 \mathrm{~cm}$ en el lóbulo superior del pulmón izquierdo, cuya histopatología fue de un adenocarcinoma mal diferenciado T2aNOMO etapa clínica IB. La espirometría mostró un FEV de 2.9 litros. Se organizó una sesión clínica multidisciplinaria en la que se plantearon las siguientes preguntas, que se formularon de acuerdo al sistema PICO.

1. En pacientes con valvulopatía candidatos a tratamiento quirúrgico y resección de cáncer de pulmón, ¿el no corregir la cardiopatía, se asocia a un incremento en la mortalidad perioperatoria?

2. En pacientes con valvulopatía candidatos a tratamiento quirúrgico y resección de cáncer de pulmón, ¿si se operan ambas patologías durante el mismo evento quirúrgico, habrá un incremento en la mortalidad o el sangrado perioperatorio?

3. En pacientes con valvulopatía candidatos a tratamiento quirúrgico y resección de cáncer de pulmón, ¿el realizar la cirugía en dos tiempos por la misma incisión se asocia a complicaciones técnicas? 
4. En pacientes con valvulopatía candidatos a tratamiento quirúrgico y resección de cáncer de pulmón, ¿el operar ambas patologías durante el mismo evento quirúrgico, pondrá en riesgo la sobrevida a largo plazo?

Se optó por dar el tratamiento sincrónico, que consistió en cambio valvular aórtico con prótesis mecánica y de lobectomía superior izquierda secuencial y en bomba de circulación extracorpórea (BCE) a través de una incisión medio esternal, logrando con ello una adecuada recuperación del paciente sin complicaciones mayores y con una sobrevida hasta la fecha de ocho años sin recidiva tumoral y sin problemas cardíacos, resultando finalmente en un tumor en etapa clínica IIB clínicamente curado.

\section{MATERIAL Y MÉTODOS}

Se realizó una búsqueda de información en PubMed en la que se incluyeron las siguientes palabras MESH en forma entrecruzada: cardiac surgery, lung cancer surgery, valvuloplasty, cardiac valve replacement, lobectomy y wedge lung resection, que se publicaron en los últimos 35 años, publicados en inglés, francés, alemán o español, restringido a artículos originales. Se realizó una evaluación de la calidad y confidencia de los manuscritos de acuerdo con el sistema de clasificación de GRADE, ${ }^{4}$ para tener un soporte metodológico de las conclusiones. Dicho sistema consiste en clasificar las evidencias en cuatro grados según la presencia o ausencia de: sesgos, inconsistencia, imprecisión, dosis-respuesta, tamaño del efecto y confusores (tabla 1). Después, los manuscritos encontrados fueron ordenados según la pregunta que podían contestar. Finalmente, se construyó una tabla con un resumen de los pacientes que se lograron extraer de los reportes de caso.

\section{RESULTADOS}

Se encontraron nueve artículos, todos ellos reportes de caso, el mayor con 79 pacientes. Siete de nueve fueron clasificados con la escala de GRADE: muy baja calidad y dos con calidad baja por carecer de grupo control. El total de pacientes reportados con cirugía cardíaca fue de 189; sin embargo, solamente 32 pacientes se sometieron a cirugía valvular. La mayoría de los estudios carecía de las especificaciones mínimas del diagnóstico y seguimiento de los casos reportados (tabla 2).

Pregunta 1: En pacientes con valvulopatía candidatos a tratamiento quirúrgico y resección de cáncer de pulmón, ¿el no corregir la cardiopatía, se asocia a un incremento en la mortalidad perioperatoria?

En un estudio clásico del tratamiento quirúrgico de cáncer de pulmón de células no pequeñas, con base en las observaciones realizadas en 621 pacientes se esta-

Tabla 1. Sistema GRADE para evaluar la calidad de las evidencias.

\begin{tabular}{|c|c|c|c|c|}
\hline Diseño & Calidad por diseño & Menor si: & Mayor si: & Calidad final \\
\hline Ensayo clínico aleatorizado & Alta & $\begin{array}{c}\text { Sesgo } \\
\text { Serio }-1 \\
\text { Muy serio -2 } \\
\text { Inconsistencia } \\
\text { Seria -1 } \\
\text { Muy seria -2 }\end{array}$ & $\begin{array}{c}\text { Efecto } \\
\text { Grande }+1 \\
\text { Muy grande }+2 \\
\text { Dosis respuesta } \\
\text { Presente }+1\end{array}$ & Alta +4 \\
\hline Estudio observacional & 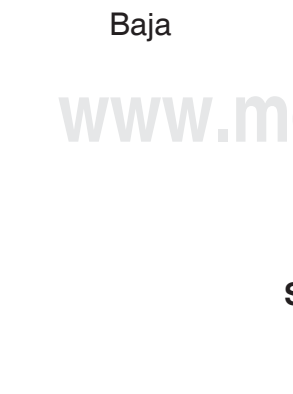 & $\begin{array}{l}\text { Falta de dirección } \\
\text { Seria }-1 \\
\text { Muy seria }-2 \\
\text { Imprecisión } \\
\text { Seria }-1 \\
\text { Muy seria -2 } \\
\text { Sesgo de publicación } \\
\text { Probable -1 } \\
\text { Muy probable -2 }\end{array}$ & $\begin{array}{l}\text { Elimina confusores } \\
\text { Que reducen el efecto }+1 \\
\text { Que muestran efecto } \\
\text { espurio }+1\end{array}$ & Muy bajo +1 \\
\hline
\end{tabular}

Adaptada de Balshem $\mathrm{H}$, et al. GRADE guidelines: 3 . Rating the quality of evidence. ${ }^{4}$ 


\begin{tabular}{|c|c|c|c|c|c|c|c|c|c|c|c|c|c|c|c|c|c|c|}
\hline & 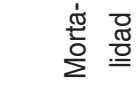 & 으 & & & & & & & & & & ఉ & ఉ & 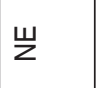 & & & & \\
\hline & 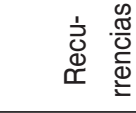 & 2 & & & & & & & & & & $\frac{\infty}{\omega^{\infty}}$ & & 岀 & & & & \\
\hline & 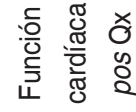 & 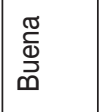 & & & & & & & & & & & & 岁 & & & & \\
\hline & 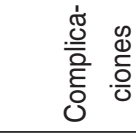 & 운 & $\sum_{1}$ & zo & 采 & & & & 2 & $\frac{\overleftarrow{c}}{\omega}$ & & & & 㞱 & 凹 & 产 & 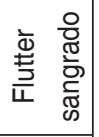 & 윯 \\
\hline த் & 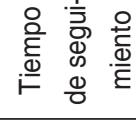 & $\stackrel{\sim}{\sim}$ & $\sigma$ & 0 & & & & & ळ) & $\wedge$ & & F & $\mathbb{\infty}$ & 岂 & & & & $\begin{array}{l}\text { ष्य } \\
\text { o } \\
0 \\
\text { 2 }\end{array}$ \\
\hline $\begin{array}{l}1 \\
0 \\
0 \\
0 \\
0 \\
0\end{array}$ & 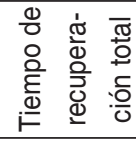 & T & $\stackrel{D}{\sim}$ & ㅁ & $\stackrel{\nabla}{ \pm}$ & & & & ले & 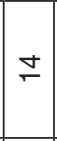 & & ষ্ল্ল & & 㞱 & $\stackrel{d}{\mathbf{N}}$ & $\stackrel{2}{\leftarrow}$ & $\stackrel{\bullet}{\check{0}}$ & \\
\hline$\sum_{0}^{\infty}$ & 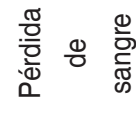 & $\begin{array}{l}\vec{E} \\
\dot{8} \\
\delta\end{array}$ & & & 员 & & & & & & & & & 㞱 & 品 & ட̊ำ & రి & \\
\hline $\begin{array}{l}0 \\
0 \\
0 \\
\frac{0}{0} \\
\stackrel{0}{0} \\
\frac{0}{0}\end{array}$ & 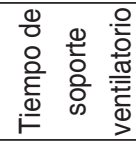 & $\frac{c}{\underline{L}}$ & $\stackrel{ᄃ}{\mathbb{N}}$ & $\stackrel{\check{c}}{\stackrel{c}{\simeq}}$ & 0 & & & & & & & & & 㞱 & $\stackrel{ }{ \pm}$ & $\sigma$ & $\infty$ & \\
\hline 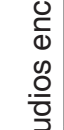 & 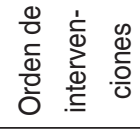 & $\begin{array}{l}\frac{0}{x} \\
\stackrel{-}{-}\end{array}$ & 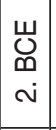 & & 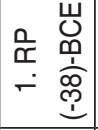 & 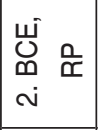 & 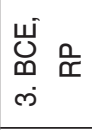 & 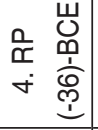 & & & & 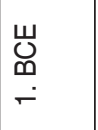 & & 岁 & & & & 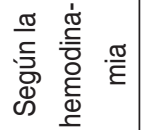 \\
\hline $\begin{array}{l}\overline{1} \\
0 \\
\infty \\
0 \\
0\end{array}$ & 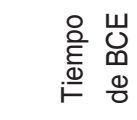 & $\stackrel{\stackrel{n}{\circ}}{\rightleftharpoons}$ & 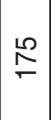 & $\stackrel{\widetilde{m}}{\sim}$ & & & & & & & & న & & 岂 & & & & 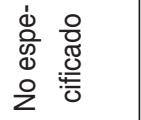 \\
\hline 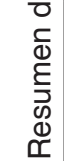 & 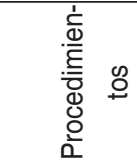 & 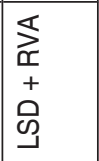 & 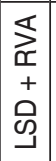 & 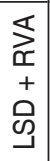 & $\stackrel{\Re}{\gtrless}$ & & & & 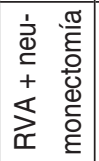 & $\underset{\pi}{\mathbb{x}}$ & $\begin{array}{l}\sum_{j} \\
+ \\
\vdots \\
\mathbb{1}\end{array}$ & $\begin{array}{l}\stackrel{5}{\circledR} \\
+ \\
\Phi\end{array}$ & 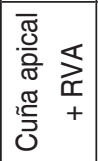 & $\stackrel{\pi}{x}$ & $\sum_{\mathbb{1}}$ & $\sum_{\Perp \Perp}$ & $\stackrel{\mathbb{3}}{\mathbb{1}}$ & 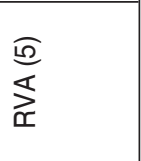 \\
\hline $\begin{array}{l}\text { N } \\
\frac{\pi}{0} \\
\frac{0}{\sigma}\end{array}$ & $\sum_{1}$ & $\begin{array}{l}\text { Oे } \\
\frac{0}{0} \\
\frac{0}{0} \\
\underline{=}\end{array}$ & 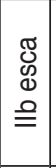 & $\begin{array}{l}\stackrel{\circ}{\bar{m}} \\
\frac{0}{0} \\
\stackrel{\rho}{=}\end{array}$ & 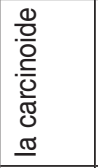 & & & & $\underline{\square}$ & 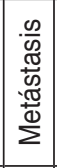 & & $\begin{array}{l}\text { ర్ } \\
0 \\
0 \\
\text { ల }\end{array}$ & 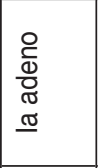 & 㞱 & $\begin{array}{l}0 \\
\stackrel{0}{0} \\
\frac{0}{0} \\
0 \\
=\end{array}$ & 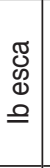 & 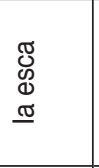 & $\stackrel{\infty}{Z}$ \\
\hline & $\begin{array}{l}\text { 晏 } \\
\text { 寽 } \\
\text { d }\end{array}$ & $\begin{array}{l}\frac{0}{0} \\
\frac{0}{8} \\
3 \\
\sum\end{array}$ & & & $\begin{array}{l}\frac{0}{\sqrt{8}} \\
\frac{0}{3} \\
\frac{3}{2}\end{array}$ & & & & 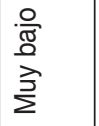 & & $\begin{array}{l}\frac{0}{\sqrt{8}} \\
\frac{0}{3} \\
\frac{3}{2}\end{array}$ & $\begin{array}{l}\frac{0}{0} \\
\frac{0}{8} \\
3 \\
\sum \\
\sum\end{array}$ & 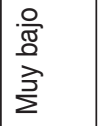 & 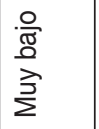 & & & & . \\
\hline & 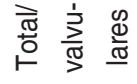 & $\frac{m}{b n}$ & & & $\underset{⿱}{\rightleftarrows}$ & & & & ले & & $\stackrel{ \pm}{t}$ & $\bar{\sigma}$ & 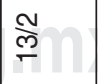 & $\stackrel{N}{\stackrel{N}{N}}$ & & & & $\stackrel{\infty}{\circ}$ \\
\hline & $\begin{array}{l}\frac{\pi}{0} \\
\text { d } \\
4\end{array}$ & ঃ̊̀ & & & ¿্ণ & & & & ષ્ণ & & ర్ & ర్ల & $\stackrel{\infty}{\stackrel{\circ}{\circ}}$ & ֻூ & & & & ஜூ \\
\hline & 产 & 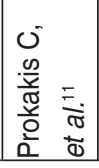 & & & 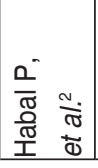 & & & & 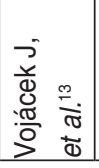 & & 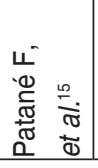 & 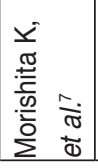 & 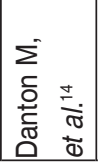 & 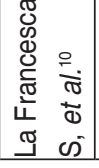 & & & & 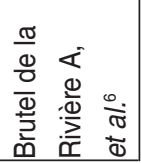 \\
\hline
\end{tabular}




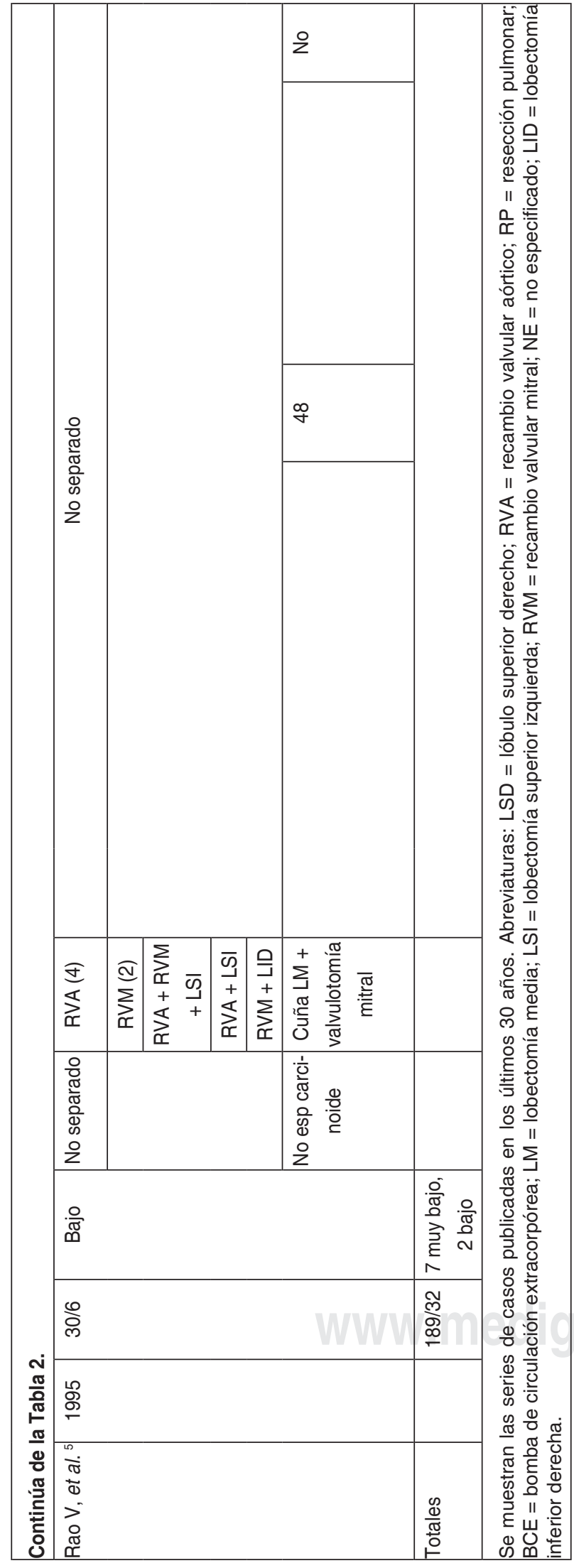

blece que la presencia de enfermedad arterial coronaria no corregida así como la clasificación de ASA 3 y 4, son factores de riesgo que incrementan la mortalidad; sin embargo, en este trabajo no se habla específicamente de la presencia de valvulopatía no corregida. ${ }^{3}$

Respuesta tentativa: por extrapolación no se recomienda llevar al paciente a cirugía de resección pulmonar por cáncer sin antes corregir la cardiopatía. GRADE: muy bajo.

Pregunta 2: En pacientes con valvulopatía candidatos a tratamiento quirúrgico y resección de cáncer de pulmón, ¿si se operan ambas patologías durante el mismo evento quirúrgico, habrá un incremento en la mortalidad o el sangrado perioperatorio?

Mortalidad: Rao et al., reportaron en una serie de 30 casos que la mortalidad no se incrementa al realizar ambas intervenciones durante la misma anestesia. ${ }^{5}$ Llama la atención el hecho de que el $30 \%$ de los pacientes reportados tenían un tumor benigno. Otro de los estudios con mayor número de pacientes es el de Brutel de la Rivière et al. con 79 , en el que se concluye que se puede realizar ambos procedimientos sincrónicamente sin incrementar el riesgo de mortalidad. ${ }^{6}$ Existen más estudios que obtuvieron conclusiones similares. ${ }^{5,7-13}$

Sangrado: Se puede disminuir el sangrado perioperitarorio y el riesgo de ventilación mecánica al evitar la BCE en los pacientes sometidos a ambos procedimientos en forma sincrónica. ${ }^{14}$

Respuesta: Con base en nueve series de casos sin grupo control, se hace evidente que en múltiples instituciones se realiza este tipo de procedimientos en forma combinada sin incremento de la mortalidad, así como sangrado mínimo; GRADE: muy bajo.

Pregunta 3: En pacientes con valvulopatía candidatos a tratamiento quirúrgico y resección de cáncer de pulmón, ¿el realizar la cirugía en dos tiempos por la misma incisión se asocia a complicaciones técnicas?

El estudio de Habal et al., ${ }^{2}$ con 11 pacientes operados en forma independiente, nos transmite que se presentaron dificultades para la disección al entrar por la misma incisión, así como del manejo perioperatorio de los anticoagulantes; GRADE: muy bajo.

A su vez, se ha encontrado que el sangrado perioperatorio puede ser menor si se comienza realizando la resección pulmonar antes de meter al paciente a bomba de circulación extracorpórea. ${ }^{15}$ GRADE: muy bajo. 
La lobectomía inferior izquierda es el procedimiento más complicado para ser realizado por estereotomía media; aunque existen alternativas, como la apertura del pericardio y la prolongación de la apertura del espacio intercostal para hacerla más fácil. ${ }^{11,16}$ GRADE: muy bajo.

Pregunta 4: En pacientes con valvulopatía candidatos a tratamiento quirúrgico y resección de cáncer de pulmón, ¿el operar ambas patologías durante el mismo evento quirúrgico, pondrá en riesgo la sobrevida a largo plazo?

En forma clásica se ha tenido miedo por realizar ambos procedimientos en forma conjunta debido al reporte de Miller et al., quienes compararon dos grupos: uno de 30 pacientes operados en forma simultánea contra $15 \mathrm{pa}-$ cientes operados en forma secuencial, encontrando una ventaja en la sobrevida a cinco años de los pacientes operados en forma secuencia principalmente aquellos en estadio I. ${ }^{17}$ GRADE: bajo.

\section{DISCUSIÓN}

Estamos viviendo plenamente la era de la Medicina Basada en Evidencias, por lo que estamos obligados ética y profesionalmente a tomar nuestras decisiones, así como nuestros consejos basándonos en los reportes de la literatura médica.

El problema de las intervenciones sincrónicas en cirugía cardiotorácica es una excusa perfecta para poner en marcha este ejercicio académico. Es muy interesante cómo cambia la perspectiva de un problema clínico cuando se revisa la totalidad de los estudios que verdaderamente pueden responder a la misma. Tal es el caso de nuestro tema, ya que se han encontrado únicamente reportes de caso, mayormente de calidad metodológica de mala a muy mala.

Al final de esta revisión, tendremos que contentarnos con tomar nuestras decisiones apoyándonos en las observaciones realizadas en 32 pacientes, con igual de pobre acuciosidad en el reporte de los mismos. Así, se vuelve más interesante contrastar estos 32 pacientes con aquel estudio de Miller et al., de 1994 en el que se reportó un incremento en la mortalidad contra un grupo control al realizar ambas operaciones en forma sincrónica. El mismo autor explica lo anterior como el resultado de ciertos fenómenos de inmunosupresión asociados a la circulación extracorpórea.

Finalmente, los autores de este artículo no tenemos el soporte necesario para descartar esta teoría.

\section{CONCLUSIÓN}

Existe una marcada tendencia a realizar ambos procedimientos en forma sincrónica observándose buenos resultados a corto y largo plazo (incluyendo nuestro caso presentado). Existe una aparente creencia que lo anterior está siendo realizado con un fuerte soporte científico; lo cual en virtud de los resultados de nuestra revisión apunta más hacia una falta de verdaderos estudios comparativos y de fundamento en las ciencias básicas.

\section{REFERENCIAS}

1. INEGI. Estadísticas a propósito del Día Mundial Contra el Cáncer. México: INEGI; 2014. p. 1-11.

2. Habal P, Simek J, Lonský V, Novotný J. Possibilities of combined surgical treatment of lung tumours and heart diseases. Acta Medica (Hradec Kralove) 2006;49(3):175181.

3. Licker $\mathrm{M}$, de Perrot $\mathrm{M}$, Höhn L, et al. Perioperative mortality and major cardio-pulmonary complications after lung surgery for non-small cell carcinoma. Eur J Cardiothorac Surg 1999;15(3):314-319.

4. Balshem $\mathrm{H}$, Helfand $\mathrm{M}$, Schünemann $\mathrm{HJ}$, et al. GRADE guidelines: 3. Rating the quality of evidence. J Clin Epidemiol 2011;64(4):401-406. doi: 10.1016/j.jclinepi.2010.07.015.

5. Rao V, Todd TR, Weisel RD, et al. Results of combined pulmonary resection and cardiac operation. Ann Thorac Surg 1996;62(2):342-346.

6. Brutel de la Rivière $A$, Knaepen $P$, van Swieten $H$, Vanderschueren R, Ernst J, van den Bosch J. Concomitant open heart surgery and pulmonary resection for lung cancer. Eur J Cardiothorac Surg 1995;9(6):310-313.

7. Morishita K, Kawaharada N, Watanabe T, et al. Simultaneous cardiac operations with pulmonary resection for lung carcinoma. Jpn J Thorac Cardiovasc Surg 2001;49(12):685-689.

8. Irie T, Oonuki T, Kei J, Sone Y, Nitta S. Peri- and postoperative courses in patients undergoing concomitant cardiac and pulmonary operations. Nihon Kyobu Geka Gakkai Zasshi 1996;44(6):747-754.

9. Lichtenberg J, Rohác J, Tosovský J, Krska Z. One-stage surgery in concomitant heart and lung disease. Cas Lek Cesk 1992;131(17):537-538.

10. La Francesca $\mathrm{S}$, Frazier $\mathrm{OH}$, Radovancěvić $\mathrm{B}$, De Caro LF, Reul GJ, Cooley DA. Concomitant cardiac and pulmonary operations for lung cancer. Tex Heart Inst J 1995;22(4):296-300.

11. Prokakis C, Koletsis E, Apostolakis E, et al. Combined heart surgery and lung tumor resection. Med Sci Monit 2008;14(3):CS17-CS21.

12. Yokoyama T, Derrick MJ, Lee AW. Cardiac operation with associated pulmonary resection. J Thorac Cardiovasc Surg 1993;105(5):912-916. 
13. Vojácek J, Hlubocký J, Burkert J, et al. Simultaneous cardiac and thoracic operations. Zentralbl Chir 2006;131(3):200-2005.

14. Danton MH, Anikin VA, McManus KG, McGuigan JA, Campalani G. Simultaneous cardiac surgery with pulmonary resection: Presentation of series and review of literature. Eur J Cardiothoracic Surg 1998:13(6):667-672.

15. Patanè $F$, Verzini $A$, Zingarelli $E$, di Summa M. Simultaneous operation for cardiac disease and lung cancer. Interact Cardiovasc Thorac Surg 2002;1(2):69-71.

16. Cooper JD, Nelems JM, Pearson FG. Extended indications for median sternotomy in patients requiring pulmonary resection. Ann Thorac Surg 1978;26(5):413-420.
17. Miller DL, Orszulak TA, Pairolero PC, Trastek VF, Schaff HV. Combined operation for lung cancer and cardiac disease. Ann Thorac Surg 1994;58(4):989-993.

\section{$\triangle$ Correspondencia:}

Dr. Gildardo Cortés Julián

Departamento de Cirugía, Instituto Nacional de

Enfermedades Respiratorias Ismael Cosío Villegas. Calzada de Tlalpan Núm. 4502, Colonia Sección XVI. 01480, México, D.F.

Teléfono: 54871700 , ext. 5210

Fax: 005256660997

Correo electrónico: gildardounam@hotmail.com

Los autores declaran no tener conflicto de intereses. 